\title{
Effective ferroptotic small-cell lung cancer cell death from SLC7A11 inhibition by sulforaphane
}

\author{
YUKO IIDA $^{1 *}$, MAYUMI OKAMOTO-KATSUYAMA ${ }^{1 *}$, SHUICHIRO MARUOKA $^{1}$, \\ KENJI MIZUMURA ${ }^{1}$, TETSUO SHIMIZU ${ }^{1}$, SOTARO SHIKANO ${ }^{1}$, MARI HIKICHI ${ }^{1}$, \\ MAI TAKAHASHI ${ }^{1}$, KOTA TSUYA ${ }^{1}$, SHINICHI OKAMOTO ${ }^{1}$, TOSHIO INOUE ${ }^{1}$, YOKO NAKANISHI ${ }^{2}$, \\ NORIAKI TAKAHASHI ${ }^{1}$, SHINOBU MASUDA ${ }^{2}$, SHU HASHIMOTO ${ }^{1,3}$ and YASUHIRO GON ${ }^{1}$ \\ ${ }^{1}$ Division of Respiratory Medicine, Department of Internal Medicine; ${ }^{2}$ Division of Oncologic Pathology, \\ Department of Pathology and Microbiology, Nihon University School of Medicine, \\ Tokyo 173-8610; ${ }^{3}$ Shonan University of Medical Science, Kanagawa 244-0806, Japan
}

Received October 20, 2019; Accepted October 14, 2020

DOI: $10.3892 / \mathrm{ol} .2020 .12332$

\begin{abstract}
Small-cell lung cancer (SCLC) is a highly aggressive cancer with poor prognosis, due to a lack of therapeutic targets. Sulforaphane (SFN) is an isothiocyanate derived from cruciferous vegetables and has shown anticancer effects against numerous types of cancer. However, its anticancer effect against SCLC remains unclear. The present study aimed to demonstrate the anticancer effects of SFN in SCLC cells by investigating cell death (ferroptosis, necroptosis and caspase inhibition). The human SCLC cell lines NCI-H69, NCI-H69AR (H69AR) and NCI-H82 and the normal bronchial epithelial cell line, 16HBE140- were used to determine cell growth and cytotoxicity, evaluate the levels of iron and glutathione, and quantify lipid peroxidation following treatment with SFN. mRNA expression levels of cystine/glutamate antiporter xCT (SLC7A11), a key component of the cysteine/glutamate antiporter, were measured using reverse transcription-quantitative PCR, while the levels of SLC7A11 protein were measured using western blot analysis. Following the addition of SFN to the cell culture, cell growth was significantly inhibited, and cell death was shown in SCLC and multidrug-resistant H69AR cells. The ferroptotic effects of SFN were confirmed following culture with the ferroptosis inhibitor, ferrostatin-1, and deferoxamine; iron levels were elevated, which resulted in the accumulation of lipid reactive oxygen species. The mRNA and protein expression levels of SLC7A11 were significantly
\end{abstract}

Correspondence to: $\mathrm{Dr}$ Shuichiro Maruoka, Division of Respiratory Medicine, Department of Internal Medicine, Nihon University School of Medicine, 30-1 Ohyaguchi-Kamimachi, Itabashi, Tokyo 173-8610, Japan

E-mail: maruoka.shuichiro@nihon-u.ac.jp

${ }^{*}$ Contributed equally

Key words: anticancer effect, cell death, ferroptosis, small-cell lung cancer, sulforaphane lower in SFN-treated cells compared with that in the control cells $(\mathrm{P}<0.0001$ and $\mathrm{P}=0.0006$, respectively). These results indicated that the anticancer effects of SFN may be caused by ferroptosis in the SCLC cells, which was hypothesized to be triggered from the inhibition of mRNA and protein expression levels of SLC7A11. In conclusion, the present study demonstrated that SFN-induced cell death was mediated via ferroptosis and inhibition of the mRNA and protein expression levels of SLC7A11 in SCLC cells. The anticancer effects of SFN may provide novel options for SCLC treatment.

\section{Introduction}

Small-cell lung cancer (SCLC), which accounted for $\sim 15 \%$ of all cases of lung cancer in the United States in 2020 (1), is a highly aggressive lung cancer tumor. The 5-year survival rate was $<7 \%$ in the United States in 2017 (2). The survival rate is poor for a number of reasons. Firstly, SCLC is characterized by rapid growth and metastasis and the lack of early detection means few cases can be treated by surgery (3). Secondly, the initial effects of chemotherapy quickly diminish, and the majority of patients relapse within the first year, worldwide (1), developing multidrug resistance. Finally, a lack of adequate surgical tissues and re-biopsy for molecular profiling results in few therapeutic options for SCLC compared with that in non-small-cell lung cancer (NSCLC).

Sulforaphane (SFN) is an isothiocyanate derived from cruciferous vegetables, particularly broccoli sprouts (4). SFN has been reported to protect against the development of numerous types of cancer, such as liver, prostate, and colon cancer, by inhibiting phase I enzymes, that activate carcinogens, and by inducing nuclear factor erythroid 2-related factor 2 (Nrf2)-regulated genes of phase II detoxification enzymes (4-7). The anticancer effects of SFN in various types of cancer have been reported, in both in vitro and in vivo studies. For example, SFN has been reported to induce apoptotic cell death in NSCLC, as well as in pancreatic, breast and prostate cancer (8-11). Other angiogenic activities of SFN are mediated by the suppression of vascular endothelial growth factor and matrix metalloproteinase-2 (12). Antimetastatic 
effects of SFN were also achieved by inhibiting cell migration and invasion $(13,14)$. However, the anticancer effects of SFN against SCLC have not yet been fully elucidated.

Ferroptosis is a type of programmed cell death, that is non-apoptotic and has been described as an iron- and reactive oxygen species (ROS)-dependent form of regulated cell death (15). The morphological features of ferroptotic cells include alterations in the mitochondrial structure, accompanied by the absence of nuclear shrinkage and ruptured plasma membranes $(15,16)$. This process is triggered by two mechanisms. The first mechanism is the inhibition of system xc- (e.g. induced by erastin) (17). System xc- is the cystine/ glutamate antiporter that imports extracellular cystine in exchange for intracellular glutamate (18). The cystine/glutamate antiporter xCT (SLC7A11) is a key component of system xc- (19). Intracellular cystine starvation leads to the depletion of glutathione (GSH) levels and subsequent inactivation of GSH peroxidase 4 (GPX4) function (20). The second mechanism of ferroptosis is direct blocking of GPX4 [e.g. induced by Ras-selective lethal (RSL)3] (21,22). GPX4 is an enzyme that reduces lipid hydroperoxides to lipid alcohols (15). The loss of GPX4 activity leads to the formation of high lipid $\operatorname{ROS}(15,22)$. In addition, excessive iron also contributes to ferroptotic cell death by producing ROS via the Fenton reaction $(23,24)$. A previous report demonstrated that siramesine and lapatinib induced ferroptosis in breast cancer cells (25). The newly discovered 'ferroptosis' form of programmed cell death (17) may provide a novel target for cancer treatment.

The present study investigated the cell death effects of SFN treatment and demonstrated the anticancer effects of SFN in SCLC cells.

\section{Materials and methods}

Reagents. SFN (R,S-sulforaphane; cat. no. S8044) was purchased from LKT Laboratories, Inc.. SFN was dissolved in DMSO and was subsequently used for each assay. Z-vad (cat. no. 4800-520), an apoptosis inhibitor, was purchased from Medical and Biological Laboratories Co., Ltd. Necrostatin-1 (cat. no. N9037), a necroptosis inhibitor, ferrostatin-1 (cat. no. SML0583), a ferroptosis inhibitor, and deferoxamine (DFO; cat. no. D9533), an iron chelator, were purchased from SigmaAldrich (Merck KGaA). The optimum concentration of each reagent (z-vad, necrostatin-1 and ferrostatin-1) was determined as previously described $(17,26,27)$. The optimum concentration of DFO was determined from preliminary experiments (data not shown). The following concentrations were used: $\mathrm{z}$-vad, $10 \mu \mathrm{M}$; necrostatin-1, $50 \mu \mathrm{M}$; ferrostatin-1, $1 \mu \mathrm{M}$; and DFO, $10 \mu \mathrm{M}$. Amrubicinol (AMR), an anthracycline anticancer agent and active metabolite derived from amrubicin, was obtained from Dainippon Sumitomo Pharma Co., Ltd.

Cell culture. The human SCLC cell lines NCI-H69 (H69), NCI-H82 (H82) and NCI-H69AR (H69AR) were purchased from American Type Culture Collection. The H69AR cell line are cross-resistant to anthracycline analogs. The normal bronchial epithelial cell line, 16HBE14o- (16HBE) were kindly provided by Dr Gruenert (Head and Neck Stem Cell Laboratory, University of California, USA). The H69, H82 and H69AR cells were cultured in RPMI-1640 medium
(Sigma-Aldrich; Merck KGaA), supplemented with 10\% FBS (Sigma-Aldrich; Merck KGaA) and $1 \%$ penicillin-streptomycin (Nacalai Tesque, Inc.) while, the $16 \mathrm{HBE}$ cells were cultured in minimum essential medium (Sigma-Aldrich; Merck KGaA) supplemented with 10\% FBS (Sigma-Aldrich; Merck KGaA) and $1 \%$ penicillin-streptomycin (Nacalai Tesque, Inc.). The cells were maintained at $37^{\circ} \mathrm{C}$ in a humidified atmosphere with $5 \% \mathrm{CO} 2$. Stocks of these cells were prepared within five passages of receipt; cells used for experiments were passaged for $<6$ months.

MTT assay. H69 cells (5,000 cells/well) were treated with increasing concentrations $(1,5,10,20 \mu \mathrm{M})$ of SFN and seeded into each well of 96-well plates. Control cells were left untreated. Cells were incubated for 24, 48 and $96 \mathrm{~h}$ at $37^{\circ} \mathrm{C}$ in a humidified atmosphere with $5 \% \mathrm{CO}_{2}$. Cell viability was measured using an MTT Cell Viability Assay kit (cat. no. 30006; Biotium, Inc.). The signal absorbances were measured at $570 \mathrm{~nm}$ using a Multiskan ${ }^{\mathrm{TM}}$ GO Microplate Spectrophotometer (Thermo Fisher Scientific, Inc.) according to the manufacturer's instructions, and the background absorbance at $630 \mathrm{~nm}$ was subtracted.

Lactate dehydrogenase ( $L D H)$ assay. To determine if cell death was induced by SFN in the H69, H82, H69AR and 16HBE cell lines, the release of LDH into the medium was measured using a Cytotoxicity LDH Assay kit-WST (LDH assay) (Dojindo Molecular Technologies, Inc.). The cells were treated with Z-vad $(10 \mu \mathrm{M})$, necrostatin-1 $(50 \mu \mathrm{M})$, ferrostatin-1 $(1 \mu \mathrm{M})$, and DFO $(10 \mu \mathrm{M}) 1 \mathrm{~h}$ before SFN treatment at $37^{\circ} \mathrm{C}$. Each sample (5,000 cells/well) treated with SFN was incubated in a 96-well plate for 48 or $96 \mathrm{~h}$ at $37^{\circ} \mathrm{C}$, and the $\mathrm{LDH}$ assay was performed according to the manufacturer's instructions. H69 and H69AR cells $(5,000$ cells/well) treated with AMR were incubated in a 96-well plate for $96 \mathrm{~h}$ at $37^{\circ} \mathrm{C}$, and the $\mathrm{LDH}$ assay was performed. The absorbance of each sample was determined at $490 \mathrm{~nm}$, using a Multiskan GO Microplate Spectrophotometer (Thermo Fisher Scientific, Inc.). LDH release (cytotoxicity) was calculated by comparing the absorbance with maximum LDH release (value measured upon adding the lysis buffer included in the kit) of control cells (28). Representative images of the H69 cell line undergoing cell death in SFN-treated $(20 \mu \mathrm{M})$ and untreated controls were obtained using Fluorescent Cell Imager (Bio-Rad Laboratories, Inc.).

Live and dead assay. The H69 cells treated with SFN $(20 \mu \mathrm{M})$ and untreated control cells were seeded into 24-well plates, at a density of $1 \times 10^{5}$ cells/well at $37^{\circ} \mathrm{C}$ for $96 \mathrm{~h}$. Cytotoxicity was measured using a LIVE/DEAD ${ }^{\mathrm{TM}}$ Viability/Cytotoxicity kit for mammalian cell analysis (Thermo Fisher Scientific, Inc.) according to the manufacturer's instructions. Calcein-AM is retained within live cells, producing green fluorescence, whereas ethidium homodimer-1 (EthD-1) enters the dead cells with damaged membranes and binds to nucleic acids, producing red fluorescence (29). A concentration of $50 \mu \mathrm{M}$ calcein-AM and $4 \mu \mathrm{M}$ EthD-1 solution was used according to preliminary experiments (data not shown). Flow cytometry analysis was performed using a flow cytometer (Gallios; Beckman Coulter, Inc.) and analyzed with Kaluza software (v1.0; Beckman Coulter, Inc.). 
Iron assay. Intracellular levels of ferrous $\left(\mathrm{Fe}^{2+}\right)$ iron were determined using an iron assay kit from Sigma-Aldrich (cat. no. MAK025; Merck KGaA), according to the manufacturer's instructions. The $\mathrm{H} 69$ cells $\left(2 \times 10^{6}\right.$ cells), treated with $20 \mu \mathrm{M}$ SFN and untreated control cells were cultured at $37^{\circ} \mathrm{C}$ for $96 \mathrm{~h}$. The absorbance of each sample was measured at $593 \mathrm{~nm}$ using a Multiskan GO Microplate Spectrophotometer (Thermo Fisher Scientific, Inc.). The results were analyzed using Skanit Software (v3.2; Research Edition for Multiskan GO; Thermo Fisher Scientific, Inc.).

Analysis of lipid peroxidation. The H69 cells, treated with SFN $(20 \mu \mathrm{M})$ and untreated control cells, were seeded into 6or 24-well plates, at a density of $2 \times 10^{5}$ cells/well at $37^{\circ} \mathrm{C}$ for 48 , 72,96 or $120 \mathrm{~h}$. DFO $(10 \mu \mathrm{M})$ and ferrostatin-1 $(1 \mu \mathrm{M})$ were added to the cells at $37^{\circ} \mathrm{C}, 1 \mathrm{~h}$ before they were treated with SFN. Lipid peroxidation was measured using an Image-iT Lipid Peroxidation kit for live cell analysis (Thermo Fisher Scientific, Inc.) according to the manufacturer's instructions. Flow cytometry analysis was performed using a flow cytometer (FACSCalibur; BD Biosciences) and mean fluorescence intensity (MFI) was analyzed using FlowJo software (v10.7.1; BD Biosciences).

Detection of intracellular ROS. The intracellular levels of ROS were detected using a fluorescence plate reader (Infinite 200Pro MPlex; Tecan Group, Ltd.), using 6-carboxy-2',7'dichlorofluorescein diacetate dye (Thermo Fisher Scientific, Inc.), according to the manufacturer's instructions. The H69 cells $\left(3 \times 10^{5}\right.$ cells $)$, treated with $20 \mu \mathrm{M}$ SFN, and untreated control cells were cultured at $37^{\circ} \mathrm{C}$ for $4,8,12,16$ or $24 \mathrm{~h}$ before subsequent analysis.

Confocal fluorescence imaging of mitochondria. The H69 cells, treated with $20 \mu \mathrm{M}$ SFN or untreated control cells, were seeded into plates, at a density of $5 \times 10^{5}$ cells $/ \mathrm{ml}$, and incubated at $37^{\circ} \mathrm{C}$ for $96 \mathrm{~h}$. To determine the mitochondrial morphology, the H69 cells were cultured in glass-bottom dishes (AGC Techno Glass Co., Ltd.) and transfected with a modified baculovirus vector (BacMam 2.0; Thermo Fisher Scientific, Inc.) encoding mitochondria-targeted green fluorescent protein according to the manufacturer's instructions. The vector $(2 \mu \mathrm{l} / 10,000$ cells $)$ was transfected into the cells for $16 \mathrm{~h}$ at $37^{\circ} \mathrm{C}$. The cells were incubated at $37^{\circ} \mathrm{C}$ in a humidified incubator with $5 \% \mathrm{CO}_{2}$. The cell nuclei were stained with Hoechst33342 (5 $\mu \mathrm{g} / \mathrm{ml}$; Thermo Fisher Scientific, Inc.), at room temperature for $5 \mathrm{~min}$ in the dark box. Images of the cells were captured with an Olympus FV1000 confocal laser scanning microscope, using an $\alpha$ PlanApochromatic X x100/1.46 NA objective with 3X digital zoom (Carl Zeiss AG).

Reverse transcription-quantitative $(R T-q) P C R$. SFN $(20 \mu \mathrm{M}) 96 \mathrm{~h}$-treated and untreated control cells (1x10 4 cells/ sample) were seeded into 96-well plates. To investigate the changes in mRNA expression levels of SLC7A11, RT-qPCR was performed using the TaqMan ${ }^{\circledR}$ Gene Expression Cellsto- $\mathrm{CT}^{\mathrm{TM}}$ kit (Thermo Fisher Scientific, Inc.) according to the manufacturer's protocol. The following temperature conditions were used: Incubation at at $37^{\circ} \mathrm{C}$ for $60 \mathrm{~min}$ then at $95^{\circ} \mathrm{C}$ for 5 min. RT-qPCR was performed using an Applied Biosystems
7500 Fast Real-Time PCR System (Thermo Fisher Scientific, Inc.). The target mRNA was amplified using a TaqMan gene expression master mix (Thermo Fisher Scientific, Inc.) and the following TaqMan gene expression assay (probe and primer): SLC7A11, Hs00921938_m1 and GAPDH, Hs02758991_g1 (both from Thermo Fisher Scientific, Inc.).

The RT-qPCR thermocycling conditions were as follows: Initial denaturation at $50^{\circ} \mathrm{C}$ for $2 \mathrm{~min}, 95^{\circ} \mathrm{C}$ for $10 \mathrm{~min}$ and 40 cycles of $95^{\circ} \mathrm{C}$ for $15 \mathrm{sec}$ and $60^{\circ} \mathrm{C}$ for $1 \mathrm{~min}$. The expression levels of target mRNA were calculated using the $2^{-\Delta \Delta C q}$ method and normalized to those of GAPDH (30).

GSH assay. Total GSH levels in SFN (20 $\mu \mathrm{M})$-treated for $96 \mathrm{~h}$ and untreated cells $\left(5 \times 10^{6}\right.$ cells/sample) were determined using a GSSG/GSH Quantification kit (Dojindo Molecular Technologies, Inc.) according to the manufacturer's instructions. The absorbance of each sample was measured at $405 \mathrm{~nm}$ using a Multiskan GO microplate spectrophotometer (Thermo Fisher Scientific, Inc.). The glutathione concentration was obtained from the calibration curve using GraphPad Prism software (v8; GraphPad Software, Inc.). A total of three independent repeats was performed.

Western blot analysis. The $\mathrm{H} 69$ cells treated with $20 \mu \mathrm{M}$ SFN for 48, 72 or $96 \mathrm{~h}$ were subsequently prepared for analysis using western blot analysis. The whole cell lysates were collected at the indicated times and lysed using RIPA buffer [25 mM Tris-HCl (pH 7.6), 150 mM NaCl, 1\% NP-40, $1 \%$ sodium deoxycholate and $0.1 \%$ SDS]. The protein levels were quantified with a Bio-Rad Protein Assay Dye Reagent Concentrate (Bio-Rad Laboratories, Inc.), according to the manufacturer's instructions. The total proteins $(20 \mu \mathrm{g})$ were separated on 4-15\% gradient gels $\left(\right.$ Criterion ${ }^{\mathrm{TM}} \mathrm{TGX}^{\mathrm{TM}}$ Precast Gel; Bio-Rad Laboratories, Inc.) using SDS-PAGE and subsequently transferred onto PVDF membranes (Merck Millipore), then blocked with 3\% BSA (Iwai Chemicals Company) mixed with TBS-Tween-20 (0.05\%; TBST) at room temperature for $1 \mathrm{~h}$. After washing three times with TBS, in $0.1 \%$ Tween-20, the membranes were incubated overnight at $4^{\circ} \mathrm{C}$ with anti-human xCT/SLC7A11 (cat. no. ab175186; 1:1,000; Abcam) and $\beta$-actin (cat. no. 4967; 1:1,000) (Cell Signaling Technology, Inc.) antibodies diluted in TBST. The membranes were subsequently incubated at room temperature for $1 \mathrm{~h}$ with a goat anti-rabbit-HRP secondary antibody (cat. no. 7074; 1:3,000; Cell Signaling Technology, Inc.). The proteins were then detected with an Amersham ${ }^{\mathrm{TM}} \mathrm{ECL}^{\mathrm{TM}}$ Prime Western Blotting Detection Reagent (Cytiva) and quantified using ImageJ software (v1.53a; National Institutes of Health).

Detection of apoptotic cell death. The $\mathrm{H} 69$ cells ( $3 \times 10^{5}$ cells), treated with $20 \mu \mathrm{M}$ SFN for $96 \mathrm{~h}$ and untreated control cells, were subsequently prepared for detection of cellular apoptosis using flow cytometry. Apoptosis was detected by analyzing the sub-G1 peaks (DNA fragmentation). The cells were stained using a Propidium Iodide Flow Cytometry kit (Abcam), according to the manufacturer's instructions. Flow cytometry was performed using a Gallios flow cytometer and analyzed using the Kaluza (v1.0) Flow Cytometry Analysis software (both Beckman Coulter, Inc.). 
A

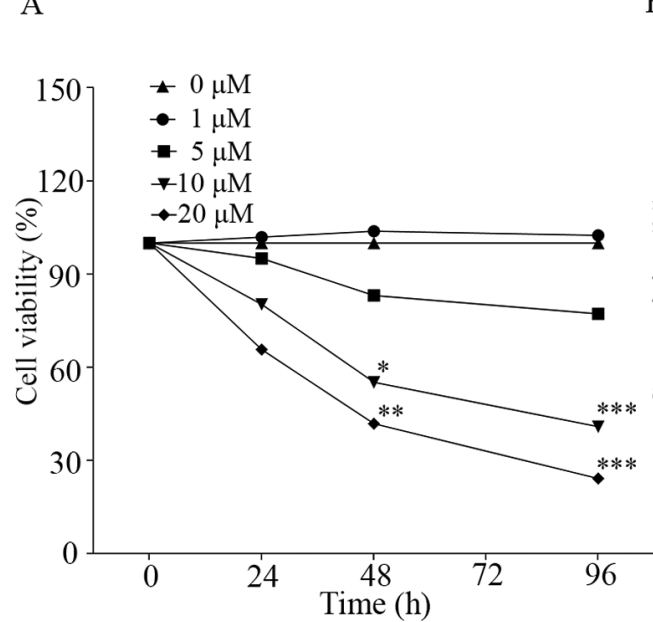

B

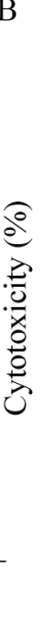

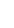

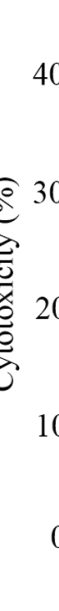

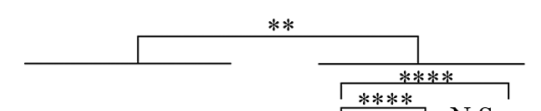

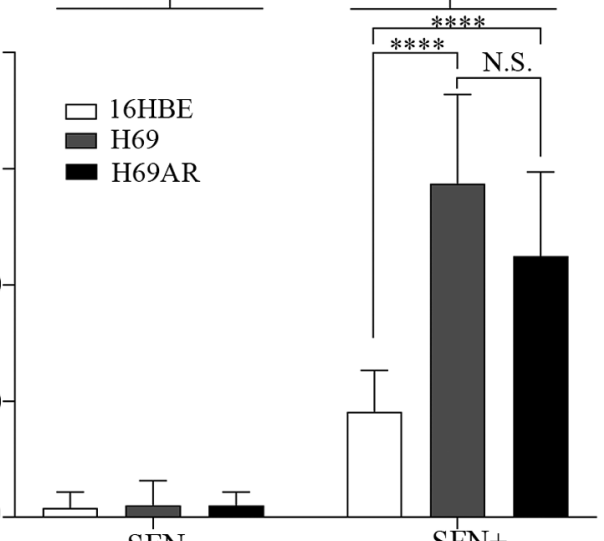

SFN-
$\mathrm{C}$
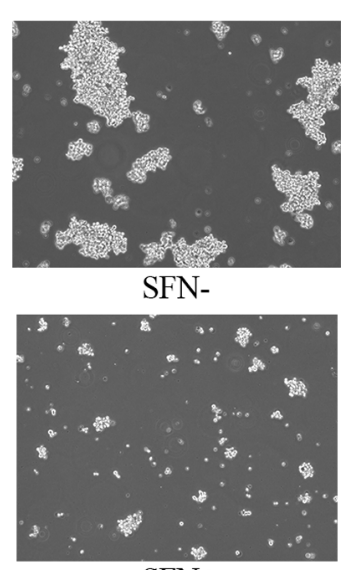

$\mathrm{SFN}+$

Figure 1. SFN induces growth inhibition and cell death in SCLC cells. (A) H69 cells were treated with $0,1,5,10$ and $20 \mu \mathrm{M}$ SFN for $0,24,48$ and $96 \mathrm{~h}$ then, cell viability was determined using a MTT assay. (B) The H69 and H69AR SCLC and 16HBE cell lines were treated with $20 \mu \mathrm{M}$ SFN for 96 h then, cell death was determined using a lactate dehydrogenase assay. (C) Representative images of H69 cells in the presence or absence of SFN (20 $\mu$ M, 96 h) treatment. Magnification, $x 20$. Data are presented as the mean $\pm \mathrm{SD}(\mathrm{n} \geq 3) .{ }^{*} \mathrm{P}<0.05,{ }^{* *} \mathrm{P}<0.01,{ }^{* * *} \mathrm{P}<0.001,{ }^{* * * *} \mathrm{P}<0.0001$. SFN, sulforaphane; SCLC, small cell lung cancer; N.S., not significant.

Statistical analysis. Data are presented as the mean \pm SD $(n \geq 3)$. The statistical significance of differences between two groups was determined using a paired or unpaired Student's t-test or Mann-Whitney U test. The statistical significance of differences among $>2$ groups was determined using one-way or two-way ANOVA followed by Tukey's multiple comparisons post hoc test. The statistical analysis was performed using GraphPad Prism software (v8; GraphPad Software, Inc.). $\mathrm{P}<0.05$ was considered to indicate a statistically significant difference; $\mathrm{P}<0.01, \mathrm{P}<0.001$ and $\mathrm{P}<0.0001$ were considered as further thresholds of significance.

\section{Results}

SFN inhibits growth and induces cell death in the SCLC cells. The effect of SFN on growth inhibition of the H69 SCLC cells was investigated using a MTT assay (Fig. 1A). Significant inhibition of cell growth was observed in H69 cells treated with $>10 \mu \mathrm{M}$ SFN over $48 \mathrm{~h}$ compared with that in the control group.

The cell death effects of SFN in the SCLC H69 and H69AR, and the normal bronchial epithelial cell line, 16HBE cell lines were initially observed to occur in a dose-dependent manner (data not shown). The cell death effects (cytotoxicity) of SFN $(20 \mu \mathrm{M})$ in the H69, H69AR and 16HBE cell lines, after $96 \mathrm{~h}$ were measured using LDH assays (Fig. 1B). A significant cell death effect was shown in the SCLC and $16 \mathrm{HBE}$ cell lines following $20 \mu \mathrm{M}$ SFN treatment compared with that in the untreated cells. However, the cell death effect in the 16HBE cell line was significantly less compared with that in the SCLC cell lines. Furthermore, the cell death effect was not significantly different between the H69 and H69AR cell lines. Similar results were observed using the SCLC H82 cell line. Significant inhibition of cell growth was observed at $24 \mathrm{~h}$ following $>20 \mu \mathrm{M}$ SFN treatment, at $48 \mathrm{~h}$ with $>10 \mu \mathrm{M}$ SFN treatment, and at $96 \mathrm{~h}$ with $>5 \mu \mathrm{M}$ SFN treatment. Cell death effects of SFN in H82 small cell lung cancer cells were observed to occur in a dose-dependent manner (Fig. S1). Fig. 1C shows representative images of H69 cell death in SFN-treated $(20 \mu \mathrm{M})$ and untreated controls from the LDH assay experiments.

Flow cytometric cell sorting analysis of the live and dead assays demonstrated that cell death was significantly induced in SFN-treated (20 $\mu \mathrm{M}$; $96 \mathrm{~h})$ SCLC cells compared with that in the untreated control cells (Fig. S2).

SFN exhibits anticancer effects against SCLC cells via induction of ferroptosis. Apoptosis, necroptosis and ferroptosis were investigated as potential cell death mechanisms caused by SFN treatment in the SCLC cells using the cell death inhibitors, z-vad, necrostatin-1 and ferrostatin-1. The results demonstrated that ferrostatin-1, which inhibits ferroptosis, significantly inhibited the effects of SFN-induced cell death. However, necrostatin-1, which inhibits necroptosis, and z-vad, which is a caspase inhibitor, did not inhibit SFN-induced cell death (Fig. 2A). In the sub G1 peak assay, the percentage of cells in the sub G1 phase $(<2 \mathrm{~N})$, in the cells treated with SFN was $6.56 \%$ compared with that in the control group $2.73 \%$ (Fig. S3).

Ferroptosis is a type of iron-dependent cell death and is known to be inhibited by the iron chelator, DFO (17). The intracellular levels of $\mathrm{Fe}^{2+}$ were significantly elevated in SFN-treated cells compared with that in untreated cells at $96 \mathrm{~h}$ (Fig. 2B). It was next determined whether DFO inhibited cell death induced by $\mathrm{SFN}$ in the H69 cell line. SFN-induced cell death was significantly decreased in DFO-treated cells compared with that in the control cells (Fig. 2C).

Ferroptosis is characterized by cell death leading to the accumulation of lipid peroxidation (17). The effects of SFN on lipid peroxidation in the H69 cells were determined using FACS analysis. MFI was significantly increased in SFN-treated cells compared with that in the untreated cells at $96 \mathrm{~h}$ (Figs. 2D and S4A). Maximum increase in the production 
A

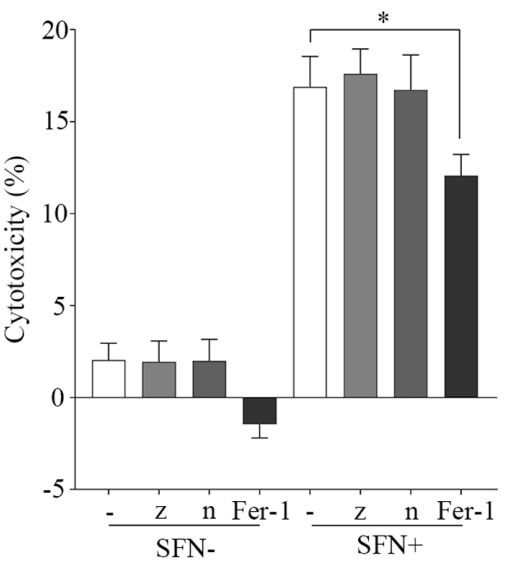

B

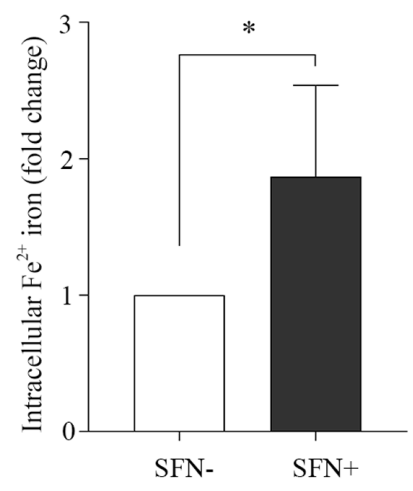

C

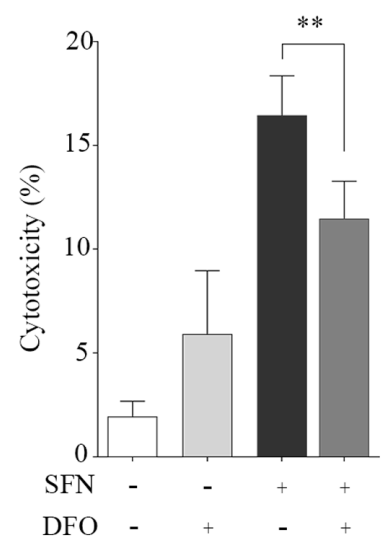

D
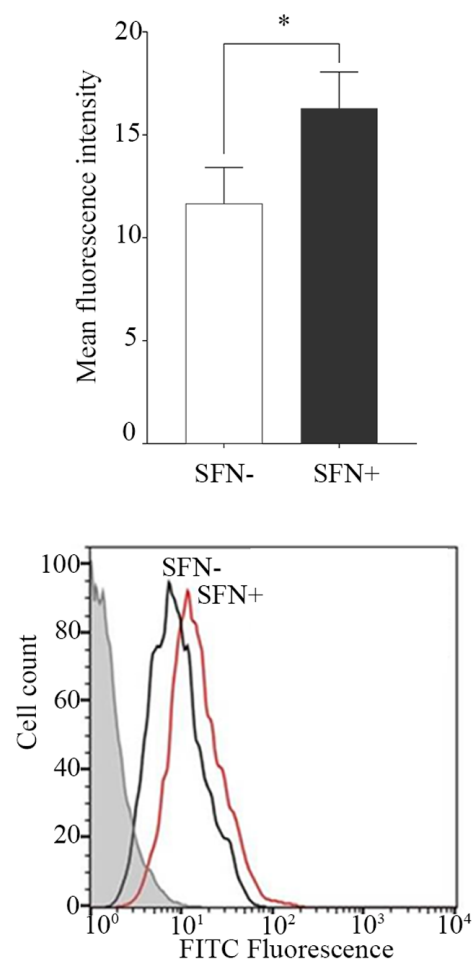

E
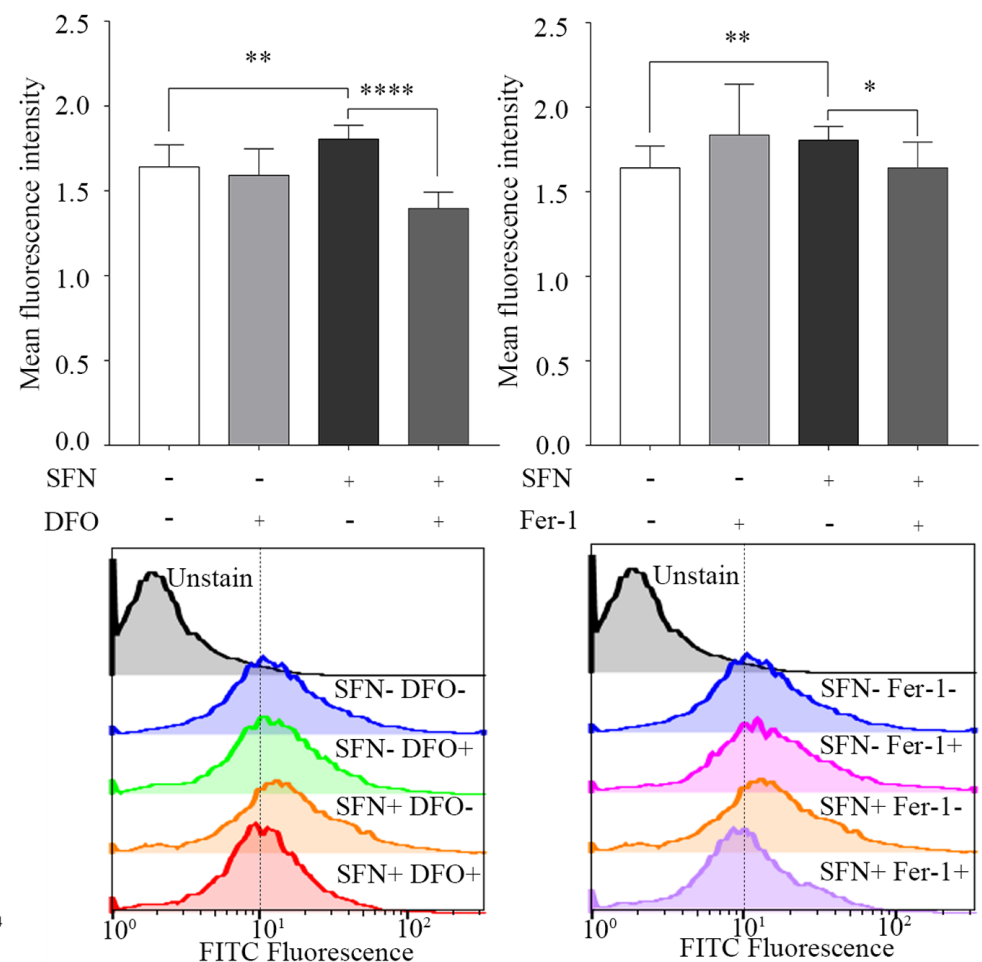

Figure 2. SFN induces cell death by ferroptosis. H69 cells were treated with SFN (20 $\mu \mathrm{M})$ for $96 \mathrm{~h}$ or untreated, as a control. (A) Inhibition of cell death by SFN using inhibitors of apoptosis, necroptosis and ferroptosis. Cell death was investigated using a LDH assay. (B) Intracellular levels of Fe ${ }^{2+}$ were determined using an iron assay and the 2 groups were statistically analyzed. (C) Inhibition of SFN-induced cell death in the presence of DFO (10 $\mu \mathrm{M}$; $1 \mathrm{~h}$ ). Cell death was investigated using a LDH assay. (D) Lipid peroxidation by SFN in H69 cells. FITC fluorescence in SFN-treated H69 cells (red line), untreated (black line) and background controls (gray filled). Lipid peroxidation was quantified using flow cytometry. (E) Inhibition of lipid peroxidation by SFN in the presence of DFO and Fer-1. Following treatment with DFO $(10 \mu \mathrm{M})$ or Fer-1 $(1 \mu \mathrm{M})$ for $1 \mathrm{~h}$, lipid peroxidation was quantified using flow cytometry. Data are presented as the mean $\pm \mathrm{SD}(\mathrm{n} \geq 3) .{ }^{*} \mathrm{P}<0.05,{ }^{* *} \mathrm{P}<0.01,{ }^{* * * *} \mathrm{P}<0.0001$. SFN, sulforaphane; LDH, lactate dehydrogenase; DFO, deferoxamine; -, no inhibitor; $\mathrm{z}, \mathrm{z}-\mathrm{vad}$; $\mathrm{n}$, necrostatin-1; Fer-1, ferrostatin-1.

of ROS induced by SFN was observed at $12 \mathrm{~h}$ (Fig. S4B). The effect of lipid peroxidation induced by SFN was reversed by pretreatment with DFO and ferrostatin-1 (Fig. 2E). Confocal fluorescence imaging indicated that the levels of mitochondria were decreased; however, there were no changes in nuclear morphology, which are characteristic features of ferroptosis $(15,16)$ (Fig. S5).

SFN inhibits SLC7A11 expression levels. Previous studies have showed that ferroptosis was initiated by the inhibition of system xc-, which leads to a depletion of GSH levels $(17,20)$.
RT-qPCR was performed to determine the mRNA expression levels of SLC7A11, a member of system xc-, following treatment of the H69 cell line with SFN (Fig. 3A). The mRNA expression levels of $S L C 7 A 11$ were $1.00 \pm 0.21$ in the untreated control cells; however, they were $0.41 \pm 0.09$ in SFN-treated cells $(20 \mu \mathrm{M}, 96 \mathrm{~h})$. Thus, the mRNA expression levels of SLC7A11 were significantly lower in the SFN-treated cells compared with that in the untreated cells. In addition, the protein expression levels of SLC7A11 in the SFN-treated cells were significantly lower compared with that in the untreated control group (Fig. 3B). 
A

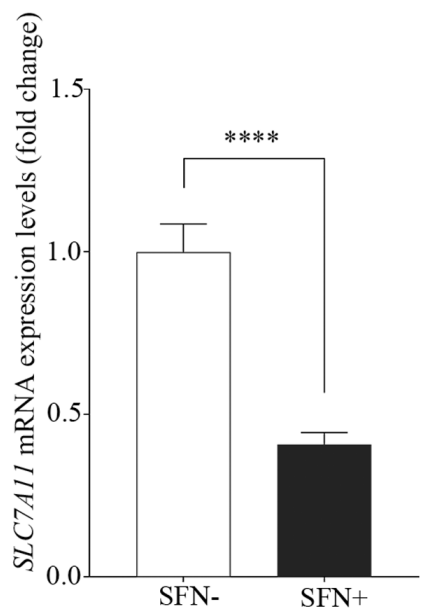

B

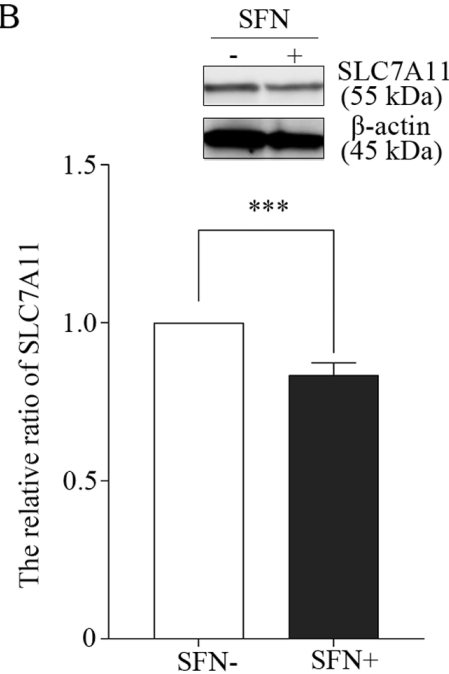

$\mathrm{C}$

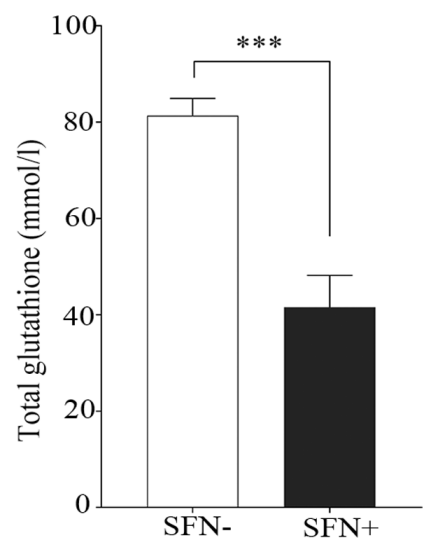

Figure 3. SFN inhibits expression levels of SLC7A11. H69 cells were treated with SFN (20 $\mu \mathrm{M})$ for $96 \mathrm{~h}$ or untreated, as a control. (A) Decreases in mRNA expression levels of SLC7A11 following SFN treatment were determined using reverse transcription-quantitative PCR. Expression levels were normalized to those of the untreated control group. Data are presented as the mean \pm SD $(n=6) .{ }^{* * * *} \mathrm{P}<0.0001$. (B) The expression levels of SLC7A11 protein were detected using western blot analysis. The band obtained at $96 \mathrm{~h}$ was quantified with ImageJ software and intensity was normalized to that of $\beta$-actin. Data are presented as the mean $\pm \mathrm{SD}(\mathrm{n}=5)$. ${ }^{* * *} \mathrm{P}<0.001$. (C) Change in glutathione concentration following SFN treatment was determined using a glutathione assay. Data are presented as the mean \pm SD $(n=9) .{ }^{* * *} \mathrm{P}<0.001$. SFN, sulforaphane; SLC7A11, cystine/glutamate antiporter $\mathrm{xCT}$.

Furthermore, intracellular total GSH levels were significantly lower in the SFN-treated H69 cells compared with that in the untreated control cells (Fig. 3C).

Cytotoxic effects of SFN in the anticancer drug-resistant H69AR cell line. The effect of SFN was analyzed in the H69AR cell lines. First, the minimum dose of AMR, that was found to be ineffective in the H69AR cell line, when compared with that in the $\mathrm{H} 69$ cell line, was investigated. At a minimum AMR concentration of $20 \mu \mathrm{M}$, the rate of cell death was significantly lower in the H69AR cell line compared with that in the H69 cell line (Fig. S6A). Thereafter, the effects of SFN were investigated in the H69AR cell line compared with that in AMR. A significant increase in induced cell death was observed in the presence of SFN $(20 \mu \mathrm{M})$ when compared with that in cells treated with AMR $(20 \mu \mathrm{M})$ in the H69AR cells (Fig. S6B). Furthermore, the SFN-induced cell death was significantly inhibited by ferrostatin-1 in the H69AR cell line (Fig. S6C).

\section{Discussion}

The present study demonstrated that SFN exhibited anticancer effects by inducing cell death via ferroptosis in the SCLC cells.

Ferroptosis is triggered by the inactivation of cellular GSH-dependent antioxidant defenses, leading to irondependent accumulation of toxic lipid ROS $(20,31)$. It has been reported that ferroptosis is an iron-dependent non-apoptotic regulated form of cell death that can be inhibited by ferrostatin-1 and the iron chelator, DFO, but not by apoptosis or necroptosis inhibitor (z-vad and necrostatin-1, respectively) $(17,20)$. However, the effect of ferroptosis in the SCLC cell is still unclear. The present study observed inhibition of SFN-induced cell death by ferrostatin-1 and DFO, GSH depletion, elevated intracellular levels of iron and the accumulation of lipid ROS, which indicated that SFN induced ferroptosis in SCLC cells.

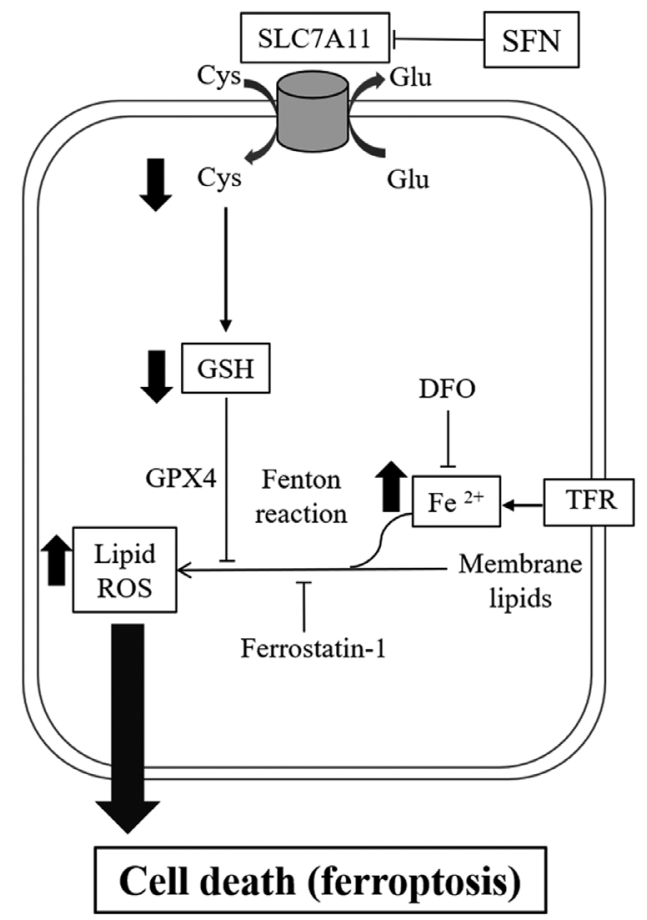

Figure 4. An overview of the SFN-induced ferroptosis pathway. SFNinduced ferroptosis observed in small cell lung cancer cells is triggered by the inhibition of SLC7A11, resulting in decreased intracellular levels of Cys and GSH. This effect leads to the accumulation of iron-dependent lipid ROS. Ferroptosis is inhibited by ferrostatin-1 and DFO. SFN, sulforaphane; SLC7A11, cystine/glutamate antiporter xCT; Cys, cysteine; GSH, glutathione; ROS, reactive oxygen species; DFO, deferoxamine; Glu, glutamate; TFR: transferrin receptor; GPX4, glutathione peroxidase 4 .

A number of mechanisms have been reported for ferroptosis inducers. For example, erastin and sorafenib induced ferroptosis by inhibition of system xc- (17). RSL3 has also been reported to directly inhibit GPX4 activity (22). The present 
study investigated the mechanisms of SFN-induced ferroptosis in 2 SCLC cell lines. According to the RT-qPCR and western blot analysis results, the mRNA and protein expression levels of SLC7A11, a component of system xc-, were significantly decreased following treatment with SFN. The present results demonstrated that inhibition of SLC7A11 expression levels, which was triggered by SFN-induced ferroptosis, resulted in decreased intracellular cystine and GSH and iron-dependent lipid peroxidation (Fig. 4).

Cytotoxic chemotherapy (e.g. cisplatin, etoposide and amrubicin) is currently a standard therapy for the treatment of advanced stage SCLC (32). These agents induce apoptotic cell death in SCLC cells (33). However, these agents also induce cell death in non-malignant cells as one of their side effects, which also include severe bone marrow suppression and pneumonia $(34,35)$. Therefore, selective and specific drugs for tumor cells are required for SCLC treatment. A previous report demonstrated that SFN $(15 \mu \mathrm{M})$ selectively induced cell cycle arrest and apoptosis in cancerous prostate epithelial cells (LnCap and PC3), but not in normal prostate epithelial cells, under the same stimulation (36). SFN may exhibit different effects on cell proliferation and death between cancer cells and non-cancerous cells. It was also confirmed that the cell death activity was significantly lower in normal bronchial cells (16HBE) compared with that in SCLC tumor cells (Fig. 1B). The present results indicated that SFN may exhibit specific anticancer effects in tumor cells, but not in normal non-cancerous cells in SCLC. Numerous types of cancer cell, such as breast and prostate cancer, exhibit higher expression levels of the cystine/glutamate antiporter system xc- compared with that in non-cancerous cells $(18,37,38)$. Cystine is an essential amino acid, and its uptake from the microenvironment is required for growth and progression in cancer (18). Furthermore, excess iron has been found to contribute to tumor growth (39), and previous studies have reported that cancer cells exhibited increased expression levels of transferrin receptors and iron uptake $(40,41)$. A previous study reported that serum iron levels were elevated in smokers (42); the majority of patients with SCLC were previous or are current smokers (2). Given the high expression levels of system xc- and excess intracellular iron, we hypothesized that cancer cells have a greater tendency to undergo ferroptosis compared with that in non-cancer cells.

Furthermore, it has previously been reported that tumor cells which exhibit an epithelial-to-mesenchymal transition (EMT)-like signature were more sensitive to ferroptosis compared with that in tumor cells, which are not sensitive (43). Zinc finger E-box binding homeobox 1, which suppresses E-cadherin, has been associated with changes in lipid accumulation and an increase in sensitivity to ferroptosis (43). SCLC is highly metastatic and develops chemoresistance via the EMT process (44). Therefore, ferroptosis may be induced in SCLC tumor cells by SFN.

The present results indicated that SFN may be used in novel anticancer strategies for SCLC. Furthermore, SFN significantly induced cell death compared to AMR in H69AR cells. SFN-induced cell death in multidrug-resistant H69AR SCLC cells was not significantly different from that induced in H69 cells. It has been reported that ferroptosis overcame cisplatin resistance in the head and neck cancer cells via pharmacological and genetic inhibition of the cystine/glutamate antiporter (45). Therefore, SFN may be a new potential adjuvant therapy for patients with SCLC, who have few effective treatment options, even after resistance to cytotoxic anticancer drugs occurs.

In the present study, the newly discovered programmed cell death 'ferroptosis' was induced by SFN treatment in SCLC cells. However, it is still unclear whether the anticancer effects of SFN was more effective in terms of cell death or cell cycle arrest. The effect on cell cycle arrest in SCLC cells with SFN treatment should be investigated further. In addition, although z-vad did not inhibit SFN-induced cell death, SFN may have induced caspase-independent apoptosis, as indicated by the increase in the percentage of SFN-treated cells in the sub-G1 phase, compared with that in the untreated control cells (Fig. S6). Further studies are required to investigate the association between SFN-induced ferroptosis and caspaseindependent apoptosis. In addition, SFN-induced detoxifying effects, that activate Nrf2-driving detoxifying genes have been previously reported (4). However, Nrf2 has been shown to be a negative regulator of ferroptosis in hepatocellular carcinoma (46) and SFN has been reported to induce different effects in prostate cancer and non-cancerous cells compared with that in Nrf2 (47). The role of Nrf2 in SFN-induced ferroptosis in SCLC cells is still unclear. Future studies will investigate the cell death effect of SFN by considering the involvement of Nrf2 in SCLC cells.

In conclusion, SFN treatment of SCLC cells led to ferroptotic cell death via the inhibition of SLC7A11, leading to decreased GSH and increased lipid ROS levels. These results indicated that SFN may be a novel potential anticancer agent for the treatment SCLC via its underlying mechanism of ferroptosis.

\section{Acknowledgements}

The authors would like to thank Professor Mariko Esumi (Division of Biochemistry, Department of Biomedical Sciences, Nihon University School of Medicine, Tokyo, Japan) for offering advice on the manuscript, and Ms. Ikuko Takeshita, Ms. Kaori Soda and Ms. Eriko Tsuboi (Division of Respiratory Medicine, Department of Internal Medicine, Nihon University School of Medicine, Tokyo, Japan) for their technical assistance.

\section{Funding}

No funding was received.

\section{Availability of data and materials}

The datasets used and/or analyzed during the current study are available from the corresponding author on reasonable request.

\section{Authors' contributions}

YI, SMar and YG conceived and designed the study. YI, TI and MOK performed the experiments. YI, MOK, KM and YN performed the statistical analysis. YI, MOK, SMar and YN wrote the paper. SS, MH, MT, KT, SO, TS, NT, SMas and SH contributed to the analysis and interpretation of data. TS, NT 
and SMas reviewed the data. GY and SH supervised the study. All authors read and approved the final manuscript.

\section{Ethics approval and consent to participate}

Not applicable.

\section{Patient consent for publication}

Not applicable.

\section{Competing interests}

The authors declare that they have no competing interests.

\section{References}

1. Tjong MC, Mak DY, Shahi J, Li GJ, Chen H and Louie AV: Current Management and Progress in Radiotherapy for Small Cell Lung Cancer. Front Oncol 10: 1146, 2020.

2. Wang S, Tang J, Sun T, Zheng X, Li J, Sun H, Zhou X, Zhou C, Zhang H, Cheng Z, et al: Survival changes in patients with smal cell lung cancer and disparities between different sexes, socioeconomic statuses and ages. Sci Rep 7: 1339, 2017.

3. Takenaka T, Takenoyama M, Inamasu E, Yoshida T, Toyokawa G, Nosaki K, Hirai F, Yamaguchi M, Shimokawa M, Seto T, et al: Role of surgical resection for patients with limited disease-small cell lung cancer. Lung Cancer 88: 52-56, 2015.

4. Clarke JD, Dashwood RH and Ho E: Multi-targeted prevention of cancer by sulforaphane. Cancer Lett 269: 291-304, 2008.

5. Myzak MC and Dashwood RH: Chemoprotection by sulforaphane: Keep one eye beyond Keap1. Cancer Lett 233: 208-218, 2006.

6. Riedl MA, Saxon A and Diaz-Sanchez D: Oral sulforaphane increases Phase II antioxidant enzymes in the human upper airway. Clin Immunol 130: 244-251, 2009.

7. Prochaska HJ, Santamaria AB and Talalay P: Rapid detection of inducers of enzymes that protect against carcinogens. Proc Natl Acad Sci USA 89: 2394-2398, 1992.

8. Kallifatidis G, Rausch V, Baumann B, Apel A, Beckermann BM Groth A, Mattern J, Li Z, Kolb A, Moldenhauer G, et al: Sulforaphane targets pancreatic tumour-initiating cells by NF-kappaB-induced antiapoptotic signalling. Gut 58: 949-963, 2009.

9. Żuryń A, Litwiniec A, Safiejko-Mroczka B, KlimaszewskaWiśniewska A, Gagat M, Krajewski A, Gackowska L and Grzanka D: The effect of sulforaphane on the cell cycle, apoptosis and expression of cyclin D1 and p21 in the A549 non-small cell lung cancer cell line. Int J Oncol 48: 2521-2533, 2016.

10. Kim SH, Park HJ and Moon DO: Sulforaphane sensitizes human breast cancer cells to paclitaxel-induced apoptosis by downregulating the NF-кB signaling pathway. Oncol Lett 13: 4427-4432, 2017.

11. Singh SV, Srivastava SK, Choi S, Lew KL, Antosiewicz J, Xiao D, Zeng Y, Watkins SC, Johnson CS, Trump DL, et al: Sulforaphane-induced cell death in human prostate cancer cells is initiated by reactive oxygen species. J Biol Chem 280 19911-19924, 2005.

12. Jackson SJ, Singletary KW and Venema RC: Sulforaphane suppresses angiogenesis and disrupts endothelial mitotic progression and microtubule polymerization. Vascul Pharmacol 46: $77-84,2007$

13. Jee HG, Lee KE, Kim JB, Shin HK and Youn YK: Sulforaphane inhibits oral carcinoma cell migration and invasion in vitro. Phytother Res 25: 1623-1628, 2011.

14. Shan Y, Zhang L, Bao Y, Li B, He C, Gao M, Feng X, Xu W, Zhang $X$ and Wang S: Epithelial-mesenchymal transition, a nove target of sulforaphane via COX-2/MMP2, 9/Snail, ZEB1 and miR-200c/ZEB1 pathways in human bladder cancer cells. J Nutr Biochem 24: 1062-1069, 2013.

15. Xie Y, Hou W, Song X, Yu Y, Huang J, Sun X, Kang R and Tang D: Ferroptosis: Process and function. Cell Death Differ 23: 369-379, 2016.
16. Gao M, Yi J, Zhu J, Minikes AM, Monian P, Thompson CB and Jiang X: Role of mitochondria in ferroptosis. Mol Cell 73: 354-363.e3, 2019.

17. Dixon SJ, Lemberg KM, Lamprecht MR, Skouta R, Zaitsev EM, Gleason CE, Patel DN, Bauer AJ, Cantley AM, Yang WS, et al: Ferroptosis: An iron-dependent form of nonapoptotic cell death. Cell 149: 1060-1072, 2012.

18. Lo M, Wang YZ and Gout PW: The x(c)- cystine/glutamate antiporter: A potential target for therapy of cancer and other diseases. J Cell Physiol 215: 593-602, 2008.

19. Jiang L, Kon N, Li T, Wang SJ, Su T, Hibshoosh H, Baer R and $\mathrm{Gu}$ W: Ferroptosis as a p53-mediated activity during tumour suppression. Nature 520: 57-62, 2015.

20. Cao JY and Dixon SJ: Mechanisms of ferroptosis. Cellular and molecular life sciences : CMLS 73: 2195-2209, 2016

21. Lu B, Chen XB, Ying MD, He QJ, Cao J and Yang B: The role of ferroptosis in cancer development and treatment response. Front Pharmacol 8: 992, 2017.

22. Yang WS, SriRamaratnam R, Welsch ME, Shimada K, Skouta R, Viswanathan VS, Cheah JH, Clemons PA, Shamji AF, Clish $\mathrm{CB}$, et al: Regulation of ferroptotic cancer cell death by GPX4. Cell 156: 317-331, 2014

23. Louandre C, Ezzoukhry Z, Godin C, Barbare J-C, Mazière J-C, Chauffert B and Galmiche A: Iron-dependent cell death of hepatocellular carcinoma cells exposed to sorafenib. Int J Cancer 133: 1732-1742, 2013

24. Shin D, Lee J, You JH, Kim D and Roh JL: Dihydrolipoamide dehydrogenase regulates cystine deprivation-induced ferroptosis in head and neck cancer. Redox Biol 30: 101418, 2020.

25. Ma S, Henson ES, Chen Y and Gibson SB: Ferroptosis is induced following siramesine and lapatinib treatment of breast cancer cells. Cell Death Dis 7: e2307, 2016.

26. Slee EA, Zhu H, Chow SC, MacFarlane M, Nicholson DW and Cohen GM: Benzyloxycarbonyl-Val-Ala-Asp (OMe) fluoromethylketone (Z-VAD.FMK) inhibits apoptosis by blocking the processing of CPP32. Biochem J 315: 21-24, 1996.

27. Degterev A, Huang Z, Boyce M, Li Y, Jagtap P, Mizushima N, Cuny GD, Mitchison TJ, Moskowitz MA and Yuan J: Chemical inhibitor of nonapoptotic cell death with therapeutic potential for ischemic brain injury. Nat Chem Biol 1: 112-119, 2005.

28. Fukami T, Iida A, Konishi K and Nakajima M: Human arylacetamide deacetylase hydrolyzes ketoconazole to trigger hepatocellular toxicity. Biochem Pharmacol 116: 153-161, 2016.

29. Ahn CH, Hong KO, Jin B, Lee WW, Jung YC, Lee H, Shin J-A, Cho S-D and Hong SD: Contribution of p38 MAPK pathway to norcantharidin-induced programmed cell death in human oral squamous cell carcinoma. Int J Mol Sci 20: 3487, 2019.

30. Livak KJ and Schmittgen TD: Analysis of relative gene expression data using real-time quantitative PCR and the 2(-Delta Delta C(T)) Method. Methods 25: 402-408, 2001.

31. Torii S, Shintoku R, Kubota C, Yaegashi M, Torii R, Sasaki M, Suzuki T, Mori M, Yoshimoto Y, Takeuchi T, et al: An essential role for functional lysosomes in ferroptosis of cancer cells. Biochem J 473: 769-777, 2016.

32. Demedts IK, Vermaelen KY and van Meerbeeck JP: Treatment of extensive-stage small cell lung carcinoma: Current status and future prospects. Eur Respir J 35: 202-215, 2010

33. Zhang JY: Apoptosis-based anticancer drugs. Nat Rev Drug Discov 1: 101-102, 2002.

34. Fisher MD and D'Orazio A; CIG Media Group: Irinotecan and cisplatin versus etoposide and cisplatin in small-cell lung cancer: JCOG 9511. Clin Lung Cancer 2: 23-24, 2000.

35. Erasmus JJ, McAdams HP and Rossi SE: Drug-induced lung injury. Semin Roentgenol 37: 72-81, 2002.

36. Clarke JD, Hsu A, Yu Z, Dashwood RH and Ho E: Differential effects of sulforaphane on histone deacetylases, cell cycle arrest and apoptosis in normal prostate cells versus hyperplastic and cancerous prostate cells. Mol Nutr Food Res 55: 999-1009, 2011.

37. Koppula P, Zhuang L and Gan B: Cystine transporter SLC7A11/ $\mathrm{xCT}$ in cancer: Ferroptosis, nutrient dependency, and cancer therapy. Protein Cell: Oct 1, 2020 (Epub ahead of print).

38. Lo M, Ling V, Wang YZ and Gout PW: The xc- cystine/glutamate antiporter: A mediator of pancreatic cancer growth with a role in drug resistance. Br J Cancer 99: 464-472, 2008.

39. Torti SV and Torti FM: Iron and cancer: More ore to be mined. Nat Rev Cancer 13: 342-355, 2013.

40. Manz DH, Blanchette NL, Paul BT, Torti FM and Torti SV: Iron and cancer: Recent insights. Ann N Y Acad Sci 1368: 149-161, 2016. 
41. Daniels TR, Bernabeu E, Rodríguez JA, Patel S, Kozman M Chiappetta DA, Holler E, Ljubimova JY, Helguera G and Penichet ML: The transferrin receptor and the targeted delivery of therapeutic agents against cancer. Biochim Biophys Acta 1820: 291-317, 2012

42. Shibata Y, Inoue S, Igarashi A, Yamauchi K, Abe S, Aida Y, Nunomiya K, Sato M, Nakano H, Sato K, et al: Elevated serum iron is a potent biomarker for spirometric resistance to cigarette smoke among Japanese males: The Takahata study. PLoS One 8: e74020, 2013.

43. Viswanathan VS, Ryan MJ, Dhruv HD, Gill S, Eichhoff OM, Seashore-Ludlow B, Kaffenberger SD, Eaton JK, Shimada K, Aguirre AJ, et al: Dependency of a therapy-resistant state of cancer cells on a lipid peroxidase pathway. Nature 547: 453-457, 2017.

44. Krohn A, Ahrens T, Yalcin A, Plönes T, Wehrle J, Taromi S, Wollner S, Follo M, Brabletz T, Mani SA, et al: Tumor cell heterogeneity in Small Cell Lung Cancer (SCLC): Phenotypical and functional differences associated with Epithelial-Mesenchymal Transition (EMT) and DNA methylation changes. PLoS One 9: e100249, 2014
45. Roh JL, Kim EH, Jang HJ, Park JY and Shin D: Induction of ferroptotic cell death for overcoming cisplatin resistance of head and neck cancer. Cancer Lett 381: 96-103, 2016.

46. Sun X, Ou Z, Chen R, Niu X, Chen D, Kang R and Tang D: Activation of the p62-Keap1-NRF2 pathway protects against ferroptosis in hepatocellular carcinoma cells. Hepatology 63: 173-184, 2016.

47. Sestili $\mathrm{P}$ and Fimognari C: Cytotoxic and antitumor activity of sulforaphane: The role of reactive oxygen species. BioMed Res Int 2015: 402386, 2015.

(i) (2) This work is licensed under a Creative Commons

Attribution-NonCommercial-NoDerivatives 4.0 International (CC BY-NC-ND 4.0) License. 